\title{
Electromagnetic interference reduction in printed circuit boards by using metamaterials: a conduction and radiation impact analysis
}

\begin{abstract}
This work aims to compare the implementation of two metamaterials for reducing electromagnetic interference (EMI) in printed circuit boards (PCBs). Specifically, complementary split-ring resonators (CSRRs) and electromagnetic bandgaps (EBGs) were etched on the ground plane of a microstrip transmission line. Both techniques were compared as EMI filters, taking into account frequency response, signal integrity, and near- and far-field radiation with regard to a reference (solid ground) board. The results of electromagnetic simulations and experimental tests show similar EMI rejection levels in both cases, but CSRRs have a significantly better signal integrity response whereas EBGs behave as lower electromagnetic radiation elements in the operation frequency band.
\end{abstract}

Keywords: complementary split-ring resonators; electromagnetic bandgaps; electromagnetic interference

\section{Introduction}

Most modern electronic systems are embedded in multilayer printed circuit boards (PCBs) exposed to increasing pollution from external and internal electromagnetic interference (EMI) sources. In recent years, several multilayer layout techniques based on metamaterial (artificial media) structures have been proposed in order to mitigate unwanted electromagnetic mode transmission. Chronologically, those solutions consist of the application of electromagnetic bandgaps (EBGs) and complementary split-ring resonators (CSRRs). EBGs are a type of metamaterials based on periodic structures whose period, $p$, is comparable to the operation signal wavelength, $\lambda$ [1]. EBGs are implemented on PCBs by modulating the wave impedance of the line or the effective dielectric constant of the surrounding medium, respectively. As a result, an interference phenomenon for electromagnetic propagating waves arises due to the Bragg effect and a low-pass filter effect can typically be achieved [2]. This 
technique has been successfully applied to reduce undesired phenomena such as simultaneous switching noise, ground bounce, EMI radiation and common-mode filtering structures [3-6].

On the other hand, CSRRs constitute sub-wavelength effective media resonators (i.e., metamaterials that satisfy the condition $\lambda<<p$ ) and can also be used as an EMImitigation design technique. Specifically, CSRRs are able to generate narrow stop-band responses that can be tuned to propagation bands that are undesired, due to their negative electric permittivity, $\varepsilon<0$ [7], in the vicinity of their resonance frequency. This rejection band can be widened by cascading several CSRRs underneath a conventional microstrip line [8]. The single-negative effective media metamaterials of which CSRRs are composed have been shown to be excellent structures for filtering and mitigating EMI issues, specifically at the conducted level [9-14]. Like EBGs, CSRRs are low-cost because no extra filtering devices or metal layers are required in their implementation (carried out by means of conventional etching techniques, typically in the PCB ground plane). In addition, CSRRs are intrinsically more compact than EBGs due to their subwavelength condition.

In this paper, both EBG and CSRR techniques are analysed in terms of rejection level, conducted scattering parameters, signal integrity and radiation impact. To the best knowledge of the authors, there are no previous works comparing the performance of EBGs and CSRRs not only in terms of conducted frequency response and signal integrity but also near- and far-field radiation impact. Although several EMI-mitigation solutions that use EBGs and CSRRs have been explored, their comparison as EMIreduction structures has not been fully addressed: for example, a near- and far-field radiation impact analysis has been carried out only for EBGs [15]. 
The paper is organized as follows. Section 2 describes the design of both twolayer EBG and CSRR microstrip test implementations, which are compared to a conventional microstrip line. In Section 3, the simulated and experimental data from the frequency response, signal integrity and radiation impact are discussed. Finally, the main conclusions of this work are drawn in Section 4.

\section{Two-layer PCB metamaterials for EMI reduction}

Three two-layer PCBs were manufactured as prototypes under test. A reference homogeneous ground microstrip line and two microstrip metamaterial PCBs based on square-shaped EBGs and CSRRs, both etched in the ground plane, were implemented using a LPKF S62 drilling machine. A two-layer topology was considered in order to minimize the cost of the study case while allowing extension to a larger number of layers. Moreover, the proposed structures were implemented in this way in order to compare the typical SI degradation of EBGs with that of CSRRs as well as their frequency responses and radiation impact from the non-homogenous ground planes. The microstrip metamaterial prototypes were designed with the following specifications: a centre stop-band frequency, $f_{o}=3 \mathrm{GHz}$, and a $\Delta=66.6 \%$ fractional bandwidth, covering a total bandwidth corresponding to 2-4 GHz.

Figure 1(a) shows the reference PCB. Basically, it emulates a potential transmission line victim and is designed in a commercial Rogers RO3010 substrate (dielectric constant, $\varepsilon_{\mathrm{r}}=10.2$; thickness, $h=1.27 \mathrm{~mm}$ ) with copper metal layers (thickness, $t=35 \mu \mathrm{m}$ ). $50 \Omega$ microstrip access lines were considered to guarantee a good matching performance in testing. All three PCBs considered have strip dimensions of width $W=1.18 \mathrm{~mm}$, length $l=15.3 \mathrm{~cm}$ and total circuit area $9.1 \times 5.1 \mathrm{~cm}^{2}$. 


\subsection{Microstrip electromagnetic bandgaps}

The EBG prototype is based on a defected ground structure (DGS) and consists of several equal squares etched in the ground plane, as shown in Figure 1(b). The perturbation dimensions were designed in order to obtain the Bragg frequency (i.e., the equivalent low-pass filter cut-off frequency) at $2 \mathrm{GHz}$ and correspond to $\mathrm{a}=1 \mathrm{x} \mathrm{b}=1 \mathrm{~cm}^{2}$ with period $p=2 \mathrm{~cm}$. If we consider the $2-4 \mathrm{GHz}$ rejection band, the considered wavelength satisfies the EBG characteristic condition (1), where $\varepsilon_{\text {eff }}$ corresponds to the effective dielectric constant [16], according to Equation (2):

$$
\begin{gathered}
\lambda=\frac{c}{f \sqrt{\varepsilon_{e f f}}} \approx p \\
\varepsilon_{e f f}=\frac{\varepsilon_{r}+1}{2}+\frac{\varepsilon_{r}-1}{2} \frac{1}{\sqrt{1+12 h / W}}
\end{gathered}
$$

\subsection{Microstrip complementary split-ring resonator lines}

Figure 1(c) illustrates the proposed CSRR ground plane as well as their main design geometric parameters. Essentially, CSRRs consist of a pairs of rings etched on the ground with apertures on opposite sides that can mainly be excited by means of an axial time-varying electric field. CSRRs behave as an LC tank that can be coupled to the microstrip host line through the transmission line capacitance. Therefore, CSSR resonance frequency is given by (3), where $L_{C}$ and $C_{C}$ correspond to the equivalent inductance and capacitance of the resonator, respectively.

$$
f_{C S R R}=\frac{1}{2 \pi \sqrt{L_{C} C_{C}}}
$$

Because there are several geometric parameters, the following design criteria were followed. The width and separation of the rings as well as the inter-resonator 
distance was fixed at the minimum resolution of the manufacturing process, $c=d=e=0.3 \mathrm{~mm}$ (Figure 1c inset). In order to obtain the specified rejection band, a simulation tuning process based on the method of moments was used to slightly detune the various resonance frequencies of each involved CSRR. Thus, a set of 29 cascaded CSRRs was implemented with variable dimensions. As a reference, the starting $2 \mathrm{GHz}$ frequency corresponds to the highest CSRR area, whose dimensions are $f=g=5.4 \mathrm{~mm}$ (Figure 1c inset), whereas the ending $4 \mathrm{GHz}$ frequency dimensions correspond to the lowest area, determined by $f=2.6 \mathrm{~mm} g=5.1 \mathrm{~mm}$.

\section{Results and discussion}

This section is devoted to the analysis of both EBG and CSRR metamaterial structures at three levels: conducted stop-band filter EMI performance, SI impact and radiation degradation due to the non-homogenous ground plane in comparison with the reference solid ground board.

The layouts of the purposed implementations were electromagnetically simulated with the method of moments using the Agilent Momentum software (2.5D) and the finite-difference time-domain (FDTD) method by means of the SEMCAD X [17] platform (3D). The EMI stop-band filter frequency response was measured using an Agilent FieldFox N9916A Microwave Analyzer in vector network analyser mode, as shown in Figure 2(a). Alternatively, the experimental setup considered to study the EMI near- and far-field radiation from the ground plane of the boards under test is illustrated in Figure 2(b) in a similar way to that proposed in [10]. A microstrip patch antenna was designed at $2.4 \mathrm{GHz}$ to capture the energy radiated from the ground plane in the nearand far-field regime, depending on distance, $r$. The antenna is located above the centre of the ground plane for each board under test. The VNA port 1 is connected to the input port of the prototype under test, whereas the VNA port 2 is connected to the patch 
antenna. The output port of the board is matched with a $50 \Omega$ load. This methodology makes it possible to capture the relative measurements of radiation (by means of the S21 parameter) in order to compare the impact of radiation inside and outside the effective stop-band of the EMI filtering for metamaterial implementations. Finally, SI performance was investigated by means of the standard eye-diagram metric. Specifically, two parameters were assessed as metrics of the eye pattern quality: maximum eye width (MEW) and maximum eye opening (MEO).

\subsection{Frequency response: $S$-parameters}

The electromagnetic simulation of the conducted S-parameters of the two-port networks corresponding to each board under test was carried out up to $6 \mathrm{GHz}$. Figure 3(a) illustrates the simulated insertion losses (S21 parameter). As expected, the results confirm the presence of the EMI stop-band frequency response according to the specifications (2-4 GHz). Although the rejection level achieved is comparable for both EBG and CSRR implementation (on the order of $40 \mathrm{~dB}$ ), a significant difference is observed concerning the return losses outside the operation frequency band. Indeed, Figure 3(b) shows that CSRR degrades the simulated S11 performance with regard to the reference microstrip PCB about $10 \mathrm{~dB}$. This return-loss degradation is increased for the EBG board about $20 \mathrm{~dB}$ below the stop-band response. Therefore, a signal integrity improvement is expected for CSRR implementation compared to EBG perturbation. This fact is attributed to the smaller impact in terms of area of the CSRR disturbance etched on the microstrip ground plane, due to its electrically smaller nature.

The S-parameter measured results are depicted in Figures 4(a) and (b), respectively. Good agreement with simulations is achieved. From experimental data, it was demonstrated that both EBG and CSRR are suitable techniques for the suppression of undesired EMI bands. In fact, the measured rejection level reached $40 \mathrm{~dB}$ and $50 \mathrm{~dB}$, 
respectively. In addition, the effective etched area was significantly lower for CSRRs than for EBGs, since the former particles are lower in electricity. The S11 trend observed in the simulations was reproduced experimentally up to $2 \mathrm{GHz}$. The test values reveal a significant matching degradation for the EBG prototype of about $|\mathrm{S} 11|<10 \mathrm{~dB}$, due to the significant area disturbance on the ground plane. The CSRR EMI filter improved the signal quality in the pass-band frequency region and an average of $|\mathrm{S} 11|<17 \mathrm{~dB}$ was obtained. Even at some frequency intervals, the return losses are close to the reference case (conventional microstrip with unaltered ground plane) which presents an average experimental value of $|\mathrm{S} 11|<20 \mathrm{~dB}$. Therefore, a significant SI improvement is expected in the CSRR EMI filter design with respect to the EBG because a minor disturbance is produced at the microstrip ground plane. This effect will be analysed in Section 3.2. At frequencies beyond the upper metamaterials EMI filter band (4-6 GHz), both EBG and CSRR present conducted insertion loss attenuation due to microwave periodicity; significant S11 degradation is also observed. These effects will be discussed in terms of total radiation and power loss in Section 3.3.

\subsection{Signal integrity performance}

According to the results analysed above, both EBG and CSRR filter structures show similar good performance in decreasing EMI at the broad-band radiofrequency range. However, the imperfect ground planes etched with slot patterns degrade the S11 parameter for the corresponding signal traces. Therefore, the impact of both implementations on SI performance should be evaluated and compared with the impact corresponding to the continuous ground plane case. In order to evaluate SI, a timedomain standard eye diagram was used. This diagram provides signal quality information in terms of noise margin (from MEO) and jitter margin (from MEW). Several cosimulations of the eye diagram of EBG and CSRR were performed. A $2^{7}-1$ 
non-return-to-zero pseudo-random bit sequence (PRBS) was injected at the input port of each board under test whereas the output port was matched with a $50 \Omega$ load. The bitsequence swing and the nominal rise/fall time were $1 \mathrm{~V}$ and $100 \mathrm{ps}$, respectively. Two cases were studied: PRBS coded at bit rates of $0.1 \mathrm{~Gb} / \mathrm{s}$ and $0.5 \mathrm{~Gb} / \mathrm{s}$, respectively. Table 1 reports the SI performance for both cases. At $0.1 \mathrm{~Gb} / \mathrm{s}$, the reference microstrip line presents $\mathrm{MEO}=984 \mathrm{mV}$ and $\mathrm{MEW}=10 \mathrm{~ns}$. The signal integrity degradation caused by the EBG ground board was about $19.1 \%$ for MEO and $0.2 \%$ for MEW. The CSRR board presented excellent results in terms of MEO and similar results concerning MEW (degradation of $2.3 \%$ and $0.4 \%$, respectively). Comparable results were obtained at $0.5 \mathrm{~Gb} / \mathrm{s}$. In this case, the reference board showed SI values of $\mathrm{MEO}=965 \mathrm{mV}$ and MEW=1.99 ns. The degradation of the MEO and MEW with respect to the solid ground board was $22.9 \%$ and $1.5 \%$ for EBG and $7.7 \%$ and $0.5 \%$ for the CSRR topology. Therefore, a significant SI improvement was achieved in the CSRR case. This result agrees with the S11 discussion detailed in the previous section. Figure 5 illustrates the eye diagram at $0.5 \mathrm{~Gb} / \mathrm{s}$ for the three boards under test, confirming those results. Good eye opening is observed for the CSRR case.

\subsection{Near- and far-field EMI radiation analysis}

Let us now analyse the EMI radiation of the proposed metamaterial implementation. In order to estimate the potential radiation frequencies, the total loss power, $P_{\text {loss, }}$, was assessed according to Equation (4):

$$
P_{\text {loss }}=1-\left|S_{11}\right|^{2}-\left|S_{21}\right|^{2}
$$

In an ideal lossless microwave network, this parameter is null, $P_{\text {loss }}=0$. However, real systems involve losses such as ohmic loss, heating and radiation. Since the impact of the two first effects can be considered low, $P_{\text {loss }}$ can be used to evaluate the radiation 
leakage of the proposed metamaterials boards. Figure 6(a) depicts the simulated electromagnetic power loss of the proposed implementations. Since no metal or dielectric losses were considered, the microstrip reference PCB presents an ideal $P_{\text {loss }}$ close to 0. It is observed that along the EMI filter operation frequency band, CSRRs are expected to radiate at a higher level than EBGs. This trend is inverted for the upper frequency range. The experimental $P_{\text {loss }}$ values confirm this behaviour (Figure 6(b)). In fact, high radiation levels are observed for the CSRR board at $2 \mathrm{GHz}$ and in the 3$4 \mathrm{GHz}$ range. Beyond $4.5 \mathrm{GHz}$, the EBG generates a higher radiation level. This behaviour is explained by the resonator nature of CSRRs, which are excited at their resonance frequency, thus generating radiation, whereas the frequency response of EBGs depends on the Bragg condition, given by:

$$
\beta=\pi / p
$$

where $\beta$ is the phase constant of the propagated signal. Therefore, the destructive interference effect is due to the periodicity of the implementation and it is expected at frequencies satisfying the condition $\lambda \sim 2 p$. In addition, the geometry of the perturbation plays a key role, according to mode-coupling theory. Indeed, the centre frequency of the EBG rejected band is given by the Fourier transform of the coupling coefficient, which depends on the geometry [18].

The radiation measurement methodology depicted in Figure 2(b) was followed. Figure 7(a) shows the EMI radiation from the boards under test in the near-field proximity at $r=1 \mathrm{~cm}$, whereas the far-field radiation was tested at $r=5$ (see Figure 7(b)). The radiation peak observed at $2.4 \mathrm{GHz}$ in all cases was due to the designed patch antenna properties. Nevertheless, the observed radiation pattern agrees with the behaviour of the measured loss power, as expected. Indeed, in the near-field regime, an average radiation reduction of about $15 \mathrm{~dB}$ was achieved for the EBG board in 
comparison with the CSRR in the EMI rejection band $(2-4 \mathrm{GHz})$. A maximum difference value of $25 \mathrm{~dB}$ was observed at $3.3 \mathrm{GHz}$. This situation was inverted at frequencies higher than $4 \mathrm{GHz}$, where EBG presented a radiation level up to $29 \mathrm{~dB}$ higher at $5.4 \mathrm{GHz}$. This trend was reproduced in the far-field regime. Those experimental results were also confirmed by means of near- and far-field electromagnetic simulation. The near-field was electromagnetically simulated at a distance of $1 \mathrm{~cm}$ from the ground plane. As an example, Figure 8 depicts the radiation level of both the EBG and CSRR topologies at $3 \mathrm{GHz}$ and $4.5 \mathrm{GHz}$ by reproducing the experimental setup condition of Figure 2(b). As expected, inside the metamaterial rejection band (i.e., at $3 \mathrm{GHz}$ ), CSRR presents a more intensive radiation level. This trend is inverted at higher frequencies, where EBG radiation is predominant (i.e., at 4.5 GHz). Those qualitative results are in good agreement with the corresponding with Figure 7(a). On the other hand, Figures 9(a) and (b) present the maximum electric field and radiated power of the three reported implementations, respectively. Again, the radiation impact of the CSRR board was clear in the resonance stop-band with an increased radiated field on the order of $14 \mathrm{~dB}$. Similar results were achieved in terms of radiated power. In the upper frequency band ( $\mathrm{f}>4 \mathrm{GHz}$ ), radiation was observed to be $20 \mathrm{~dB}$ higher for the EBG structure.

\section{Conclusion}

Two metamaterial techniques based on EBG and CSRR were studied and compared as EMI-mitigation solutions for two-layer PCBs. The novelty of this work is the analysis of the perturbed microstrip ground boards with comparable EMI-reduction levels from the point of view not only of conducted scattering parameters but also of signal integrity and near- and far-field radiation. The results demonstrate the excellent performance of CSRRs in terms of noise and jitter margin, with signal integrity degrading the MEO by 
only $7.7 \%$ with regard to a conventional microstrip transmission line in the worst-case scenario. Conversely, radiation results at the EMI filter operation frequency band reveal that EBGs behave as significantly lower electromagnetic radiation elements, with an average difference in value of $15 \mathrm{~dB}$ with respect to CSRRs.

\section{Acknowledgement}

This work was supported by the Spanish Government (Ministry of Economy and Competitiveness) under Project TEC2010-18550 and AGAUR 2009 SGR 1425. The authors would like to thank to Schmid \& Partner Engineering AG for SEMCAD X for the donation under SEMCAD for Science program.

\section{References}

1. Sievenpiper, D., Zhang, L., Broas, R., Alexopulos, N.G. and Yablonovitch, E., "High-impedance electromagnetic surfaces with a forbidden frequency band", IEEE Trans. on Microwave Theory Tech., Vol. 47, pp. 2059-2073, November 1999.

2. Shahparnia, S. and O. M. Ramahi, "Electromagnetic interference reduction from printed circuit boards using electromagnetic bandgaps", IEEE Trans. on Electromagnetic Compatibility, Vol. 46, 580-587, November 2004.

3. Lin, D.-B., K.-C. Hung, C.-T. Wu and C.-S. Chang, "A serpent bridge electromagnetic bandgap structure for suppressing simultaneous switching noise", Journal of Electromagnetic Waves and Applications, Vol. 23, 213-220, 2009.

4. Huang, C.-H. and T.-L. Wu, "Analytical design of via lattice for ground planes noise suppression and application on embedded planar EBG structures", IEEE Trans. on Components, Packaging and Manufacturing Technology, Vol. 3, 21-30, January 2013.

5. Rao, P. H. and M. Swaminathan, "A novel compact electromagnetic bandgap structure in power plane for wideband noise suppression and low radiation", IEEE Trans. on Electromagnetic Compatibility, Vol. 53, 580-587, November 2011.

6. De Paulis, F., Raimondo, L., Archambeault, B., and Connor, S. and Orlandi, A., "Compact configuration of a planar EBG based CM filter and crosstalk analysis", IEEE International Symposium on Electromagnetic Compatibility 2011, pp. 704-709.

7. Falcone, F., T. Lopetegi, J.D. Baena, E. Marqués, F. Martín and M. Sorolla, "Effective negative- $\varepsilon$ stopband microstrip lines based on complementary split ring resonators”, IEEE Microwave and Wireless Component Letters, Vol. 14, 280-282, June 2004.

8. Gil, I., R. Fernández and J. Balcells, "Analysis and characterization of coupled transmission lines loaded with complementary spiral resonators”, IET Microwave Antennas and Propagation, Vol. 5, 290-297, February 2011. 
9. Weng, L .H., S. J. Shi. X. Q. Chen, Y. C. Guo and X W. Shi, “A novel CSRRs DGS as lowpass filter”, Journal of Electromagnetic Waves and Applications, Vol. 22, 1899-1906, 2008.

10. Xiang, Q.-Y., Q.-Y. Feng and X.-G. Huang, "Bandstop filter based on complementary split ring resonators defected microstrip structure”, Journal of Electromagnetic Waves and Applications, Vol. 25, 1895-1908, 2011.

11. Bait-Suwailam, M. M. and O. M. Ramahi, "Ultrawideband mitigation of simultaneous switching noise and EMI reduction in high-speed PCBs using complementary split-ring resonators", IEEE Trans. on Electromagnetic Compatibility, Vol. 54, 389-396, April 2012.

12. Pérez, D., I. Gil, J. Gago, R. Fernández, J. Balcells, D. González, N. Berbel and J. Mon, "Reduction of EMI susceptibility in circuits based on operational amplifiers using complementary split-ring resonators”, IEEE Trans. on Components, Packaging and Manufacturing Technology, Vol. 2, 240-247, February 2012.

13. I. Gil and R. Fernández, "Differential and common-mode filters based on metamaterials resonators”, International Symposium on EMC Europe, Brugge, Belgium, September 2013.

14. I. Gil and R. Fernández, "Comparison between complementary split ring resonators and electromagnetic band-gap as EMI reduction structures”, International Symposium on EMC Europe, Wroclaw, Poland, September 2010.

15. Choi, J., D. G. Kam, D. Chung, K. Srinivasan, V. Govind, J. Kim and M. Swaminathan, "Near-field and far-field analyses of alternating impedance electromagnetic bandgap (AI-EBG) structure for mixed-signal applications”, IEEE Trans. on Advanced Packaging, Vol. 30, 180-190, May 2007.

16. Pozar, D.M., "Microwave engineering”, $4^{\text {th }}$ Edition, Wiley 2012.

17. Schmid \& Partner Engineering AG (SPEAG). SEMCAD. [Online]. Available: http://www.speag.com

18. Katsenelenbaum, B. Z., L. Mercader del Río, M. Pereyaslavets, M. Sorolla Ayza, M. Thumm, Theory of Nonuniform Waveguides: The Cross-Section Method. The Institution of Electrical Engineers, 1998. 
Tables

\begin{tabular}{|c|c|c|c|c|}
\hline \multirow{2}{*}{ Implementation } & \multicolumn{2}{|c|}{ Bit rate=0.1 Gb/s } & \multicolumn{2}{c|}{ Bit rate=0.5 Gb/s } \\
\cline { 2 - 5 } & MEW (ns) & MEO (mV) & MEW (ns) & MEO (mV) \\
\hline Reference & 10 & 984 & 1.99 & 965 \\
\hline EBG & 9.98 & 769 & 1.96 & 744 \\
\hline CSRR & 9.96 & 961 & 1.98 & 891 \\
\hline
\end{tabular}

Table 1. Comparison of signal integrity performance for the three boards under test. 


\section{Figure Captions}

Figure 1. (a) Microstrip solid ground reference board top-side and relevant dimensions. This topology is common to all three boards under test. (b) EBG ground plane (board bottom-side) and relevant perturbation dimensions. (c) CSRR ground plane (board bottom-side) and relevant particle dimensions.

Figure 2. (a) Experimental setup used to analyse the EMI conducted insertion and return losses. (b) Experimental setup used to study EMI radiation from metamaterials and homogeneous ground PCBs.

Figure 3. Electromagnetic simulation of the magnitude of (a) insertion losses and (b) return losses for the microstrip reference and the EBG ground and CSRR ground boards.

Figure 4. Experimental results of the magnitude of (a) insertion losses and (b) return losses for the microstrip reference and the EBG ground and CSRR ground boards.

Figure 5. Generated eye diagrams for a bit rate of $0.5 \mathrm{~Gb} / \mathrm{s}$. (a) Reference solid ground board. (b) EBG microstrip design. (c) CSRR microstrip design.

Figure 6. (a) Electromagnetic simulation and (b) experimental power loss for the microstrip reference and the EBG ground and CSRR ground boards.

Figure 7. Measured S21 magnitude, according to the radiation measurement setup detailed in Figure 2(b). The microstrip reference, EBG ground and CSRR ground boards were tested at different distance test points: (a) $r=1 \mathrm{~cm}$; (b) $r=5 \mathrm{~cm}$.

Figure 8. Simulated near-field radiated electric field for the EBG and the CSRR topology at $3 \mathrm{GHz}$ and $4.5 \mathrm{GHz}$.

Figure 9. (a) Simulated far-field maximum radiated electric field and (b) radiated power for the microstrip reference and the EBG ground and CSRR ground boards. 